\title{
The polymer converter for effectively connecting polymer with silica optical fibres
}

\author{
P. PURA-PAWLIKOWSKA*1, M. DUDEK ${ }^{1,2}$, R. WONKO $^{1}$, P. MARĆ $^{1}$, M. KUJAWIŃSKA $^{2}$, \\ and L.R. JAROSZEWICZ ${ }^{1}$ \\ ${ }^{1}$ Faculty of Advanced Technologies and Chemistry, Military University of Technology, \\ ul. gen. Sylwestra Kaliskiego 2, Warsaw 00-908, Poland \\ ${ }^{2}$ Institute of Micromechanics and Photonics, Warsaw University of Technology, ul. Św. A. Boboli 8, \\ Warsaw 02-525, Poland
}

\begin{abstract}
We present a micrometer-size polymer converter (microbridge) for connecting polymer optical fibres with silica fibres. The procedure of preparing such microstructure is based on a process of photopolymerization. A polymer converter grows as an extension of the fibres' cores due to a self-guiding effect of the light beam in a photopolymerizable medium. Since the polymer microbridge has refractive index greater inside than outside, such a structure works as a waveguide leading the light beam between connected optical fibres. By selection of time of light exposition to the mixture and optical power of the incident light beam, it is possible to obtain a polymer converter with very good optical and mechanical characteristics. Possibility of using polymer microbridges grown directly from the fibres' core as coupling elements between silica and polymer fibres is a good alternative for obtaining permanent coupling of such fibres.
\end{abstract}

Keywords: fibre-optic element technology, photopolymerization, fibre coupling, microstructured device.

\section{Introduction}

During the last decades an increasing interest is observed in manufacturing of different types of microstructural devices at the end-faces of optical fibres. Such elements are working as special cavity or micro-lenses. The first kind of devices physically work as a modified Fabry-Perot interferometer and use advanced technology of femtosecond laser micromachining $[1,2]$ or simple synthetic polymer microgels' technology $[3,4]$ and could be applied directly as mechanical or biochemical sensors $[5,6]$. The second one utilizes laser micromachining technology [7] and can be applied as efficient coupling between laser and fibre structures [8,9] or fibre and integrated waveguide [10,11], as well as in the near-field scanning optical microscopy [12] or could be the first step for preparation of coupling elements between different types of optical fibres. Unfortunately, the above cited few methods of such microelements' manufacturing are dedicated exclusively for silica optical fibres (SOFs).

In the same time, polymer optical fibres (POFs), considering their special properties such as high robustness, low weight, large diameter of the core, high mechanical strength,

*e-mail: paulina.pura@wat.edu.pl etc. [13], started to be willingly and widely used in optics. Moreover, there exist also economic advantages as low costs of production of such fibres. For the above reasons, POFs have found applications as a high-capacity transmission medium in short distance communications' links including such areas as local area networks [14], motorization (cars and trains), sensors' application mainly in medicine, etc. However, in order to make full use of the POFs' potential, they should be connected with the silica fibres for which there is a wide range of efficient coupling sources and detector devices. Unfortunately, it is impossible to use conventional arc and filament fusion splicers [15] or $\mathrm{CO}_{2}$ laser [16] technologies for splicing POFs with SOFs because of different melting points of such fibres. Moreover, such connection should solve two additional problems. The first is the difference between refractive index value of silica and polymer structure which influences high connection losses associated with back reflection. The second is the difference between diameter of optical beam guiding in both structures.

For the above reason, possibility of using polymer microbridges grown directly from the POF and SOF cores seems to be an interesting idea. First presented in 2001 by Bachelot et al. [17] the photopolymerization method consists of growing a polymer microstructure which is an extension of the 
optical fibre core. This method, after some modification [18], seems to be an attractive alternative for preparing a connection between two optical fibres. Generally, in this method, the light is exposed through the optical fibre to a photopolymer liquid solution positioned at the other end of the fibre. The permanent junction between different types of optical fibres can be obtained after some modification of the above method [19]. The self-written waveguide structure in a photopolymerization phenomenon results from the competition of the incident Gaussian beam diffraction and photopolymerization which tends to increase the refractive index (RI) where the light intensity is the highest [20-22]. For such approach, the polymer structure grows with the higher RI inside the material as the core region and lower RI outside as the cladding region. Various dimensions of microbridges could be gained using this method by controlling physical, as well as chemical parameters of the process [23].

In this paper we report on the process of manufacturing polymer microbridges between polymer and silica optical fibres. Although there are similar works on this topic for instance [24], in author's opinion it is the first report on preparing such polymer structures for connecting POFs with SOFs which could be a permanent efficient polymeric connection of such structures using method described previously only for SMFs [25] targeted at production of the microbridge as the sensor element [26,27], as well. Authors believe that such microstructures could find potential applications in data communications [28], sensor technology [22], and even in works according optical interconnects [29].

\section{Experimental system arrangement}

As fundamental structures, commercially available optical fibres have been chosen for an experimental investigation including Graded-Index Perfluorinated Polymer Optical Fibre GIPOF62 (ThorLabs) and multimode silica fibre MMF (Corning) which have the similar cores' diameters. The main parameters of a GIPOF62 fibre are: core diameter of $62.5 \pm 5 \mu \mathrm{m}$ with RI around 1.358, cladding diameter of $85 \pm 5 \mu \mathrm{m}$ (over cladding diameter of $490 \pm 5 \mu \mathrm{m}$ ), $\mathrm{NA}=0.185 \pm 0.015$, and attenuation above $60 \mathrm{~dB} / \mathrm{km}$ at a wavelength of $\lambda=850 \mathrm{~nm}$, as well as at $\lambda=1310 \mathrm{~nm}$. The above characteristics for MMF are: core diameter of $62.5 \mu \mathrm{m}$, RI value of about 1.48 , cladding diameter of $125 \mu \mathrm{m}, \mathrm{NA}=0.273 \pm 0.013$ and attenuation below $3.5 \mathrm{~dB} / \mathrm{km}$ at a wavelength $\lambda=850 \mathrm{~nm}$ and below $0.4 \mathrm{~dB} / \mathrm{km}$ at $\lambda=1310 \mathrm{~nm}$.

The photopolymerizable formulation is made up of three basic components: sensitizer dye (Eosin Y, disodium salt), co-initiator (MDEA - methyldiethanolamine) and multifunctional acrylate (triacrylate) monomer (PETA - penthaerythritol-triacrylate), mixed in a proper weight proportion [17]. Such mixture has high sensitivity for the wavelength in the range from $450 \mathrm{~nm}$ to $550 \mathrm{~nm}$ (maximum for $530 \mathrm{~nm}$ ) and it undergoes a free-radical photopolymerization under green laser irradiation creating a three-dimensional network of the polymer. Moreover, the physical and chemical properties of the formulation such as photopolymerization threshold energy, spectral sensitivity, and viscosity can be easily changed by simply modifying its components (changing concentration of each component or the monomer itself).

The above mixture gives a possibility for fabrication of polymer microbridges between considered optical fibres by application of the optical setup illustrated in Fig. 1. This setup consists in general of three main modules: CW semiconductor laser operated at a wavelength of $532 \mathrm{~nm}$ as a source of light for photopolymerization which energy is controlled by an optical attenuator, optical fibre coupler for dividing green laser beam into two beams led in two optical fibres and the positioning mechanisms with a camera to control the arrangement of two aligned fibres [19]. Additionally, the $622 \mathrm{~nm}$ laser has been used for photopolymerization pro-

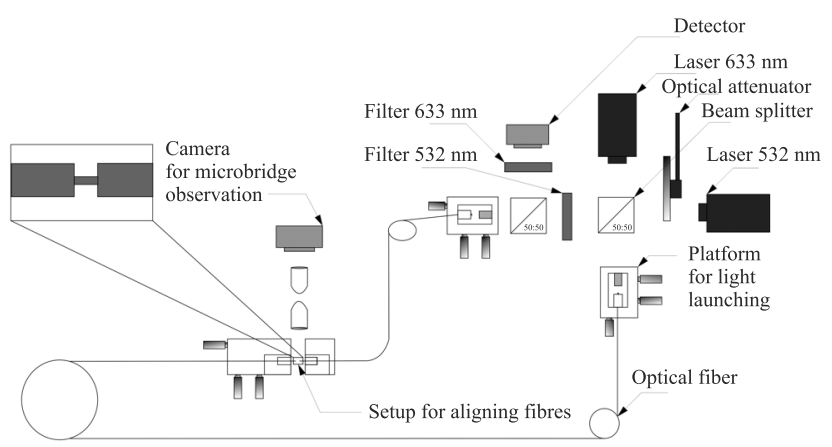

(a)

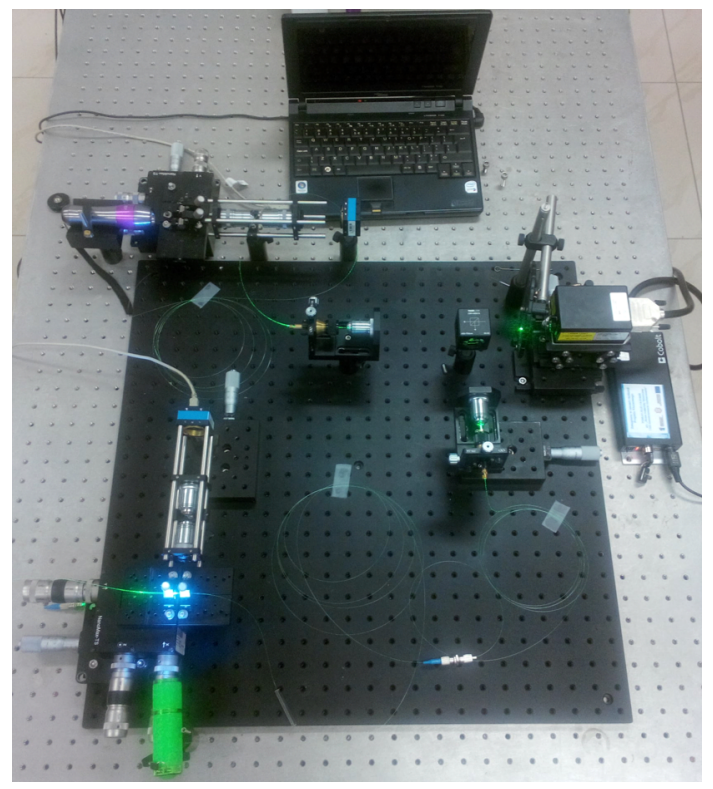

(b)

Fig. 1. General schema, as well as a view of the optical setup for the polymer microbridges fabrication. 
cess control. Process of the microbridge fabrication starts with deposition of a drop of the photopolymerizable mixture at the cleaved ends of optical fibres which are aligned coaxially with help of a visible red light. From other ends of the fibres the green light $(532 \mathrm{~nm})$ is led in. Process of photopolymerization progresses due to exposition of the mixture to the light and a polymer bridge appears as the junction between two fibres. The final structure of microbridges, their shape and width depend on the shape of guiding modes in structure, used light power, as well as exposure time [19,20, 25]. Required length of the polymer junction can be obtained by moving apart fibres to a suitable distance. After the process, the unpolymerized liquid was washed out by an alcohol.

Finally, a climate chamber VLC 7010 (Vötsch Industrietechnik) has been used for investigation of temperature characteristics of the manufactured connection in the range from $-40^{\circ} \mathrm{C}$ to $+80^{\circ} \mathrm{C}$. We investigated the changes of spectral characteristics in a wide range of wavelengths using a supercontinuum source SC450-4-AOTF (Fianium) and an optical spectrum analyzer AQ6319 (Yokogawa), as well as optical losses' changes at a wavelength of $1310 \mathrm{~nm}$.

\section{Numerical simulation of polymer microbridge properties}

The best results for the optical microbridge working as a beam converter (namely the lowest insertion and return losses), should be obtained when the bridge reproduces connected fibre structures themselves. However, in case of fibres used in this studies (SOF and POF) it was not possible, because of their different geometries as well as refractive index distributions.

In order to obtain theoretical model of light propagation from POF to SOF (fibre 1 to fibre 2) through the polymer microbridge as shown in Fig. 2a, the FDTD Solutions (by Lumerical) [30], which is a commercial 3D Maxwell equations' solver based on the Finite Difference Time Domain (FDTD) technique, has been used. Optical loss calculations have been conducted based on the methods described in Snyder and Love [31] and using mode expansion monitors a feature of FDTD Solutions. To get the insertion loss, a fraction of the field coupled into the first twenty modes of the second fibre (SOF) was calculated. Whereas to get the return loss a calculation of the fraction of the field that was reflected and coupled into the first twenty modes of the first fibre (POF) was provided.

Although the above structures are multimode, only the first twenty modes propagating in structures for a wavelength equal to $1300 \mathrm{~nm}$ are included in simulation due to the limitation of the FDTD Solutions. Moreover, we took advantage of the symmetries in the structure to reduce simu- lation time and memory requirements and we applied the uniform meshing $(\Delta \mathrm{x}=\Delta \mathrm{y}=\Delta \mathrm{z}=50 \mathrm{~nm})$ in order to reduce some of digital noise.

Model of the POF used for simulation (GIPOF62) had RI of the core changing from 1.356 to 1.342 at the core radius $(31.25 \mu \mathrm{m})$ and RI of the cladding equal to 1.342. For the used SOF structure (MMF) RI of the core changing from 1.48 in the center to 1.4504 ( $2 \%$ difference) at the core radius $(31.25 \mu \mathrm{m})$ and $\mathrm{RI}$ of the cladding was also of 1.4504 . The simulated optical microbridge was $10 \mu \mathrm{m}$ wide with the core radius of $30 \mu \mathrm{m}$ and the cladding radius of $60 \mu \mathrm{m}$. The microbridge core RI was changing from 1.52 at the center to 1.51 at the inner radius, RI of the cladding was of 1.51 , according to experimental results previously presented [22]. Beam intensity distribution in the microbridge for the above mentioned twenty modes from the POF was presented in arbitrary units in Fig. 2(a). It can be seen that the light source was located at $-0.5 \mu \mathrm{m}$, as the intensity values below this point came from reflection and were lower by the factor of 3 than the one for transmission. We can clearly see this by comparing intensity of distributions transmitted through the optical microbridge [Figs. 2(b) and 2(d)] and the one reflected from the structure [Figs. 2(c) and 2(e)]. Basing on those data we could estimate the insertion loss (IL) and the return loss (RL) to be around $0.196 \mathrm{~dB}$ and around $22.0 \mathrm{~dB}$, respectively, while the $\mathrm{IL}$ and $\mathrm{RL}$ for fundamental mode were calculated to be $0.1 \mathrm{~dB}$ and $32.271 \mathrm{~dB}$, respectively.

\section{Results and discussion}

The optimization procedure for manufacturing the polymer microbridges as a converter for effectively connecting optical fibres consisted of choosing three parameters: time of the light exposition to a photopolymerizable medium, initial power of light and length of the microbridge. Since polymer microbridges grow as the extension of the fibres' cores due to a self-guiding effect of the light beam in a photopolymerizable medium, the permanent junction between two fibres can be obtained. Such microbridge gives opportunity to cover up small shortcomings in the fibres alignment. RI of the final microbridge structure is the highest where intensity of the incident light beam is the largest. In this way a microbridge works as a waveguide with the core and the cladding [20]. By changing the first of the parameters mentioned above, the microbridge demonstrates different characteristics including proper optical losses or good mechanical strength. Different width of microbridges can be gained by controlling the output power from the fibre which is correlated with the guiding structure of light in a fibre [32]. As the output power increases, width of the microbridge increases, as well. Generally, width of a microbridge does not have any significant influence on optical losses. However, it has to be noticed that for different fibres a diameter of the bridge at the 


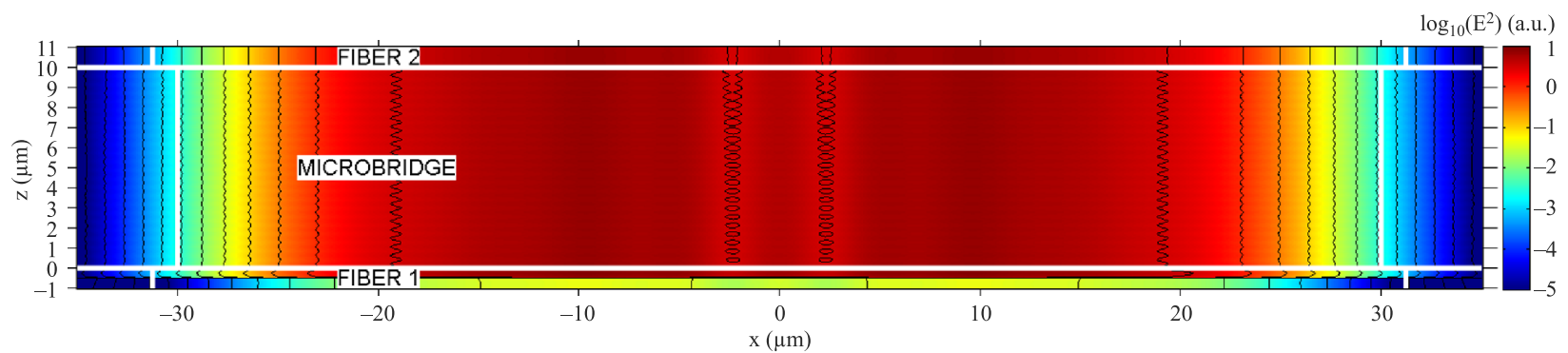

(a)

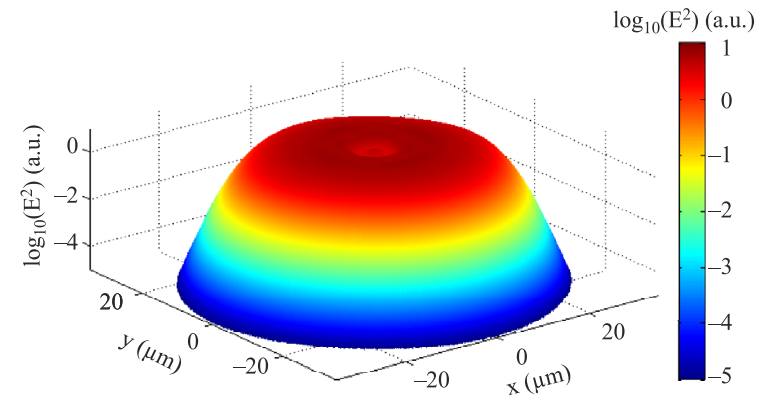

(b)

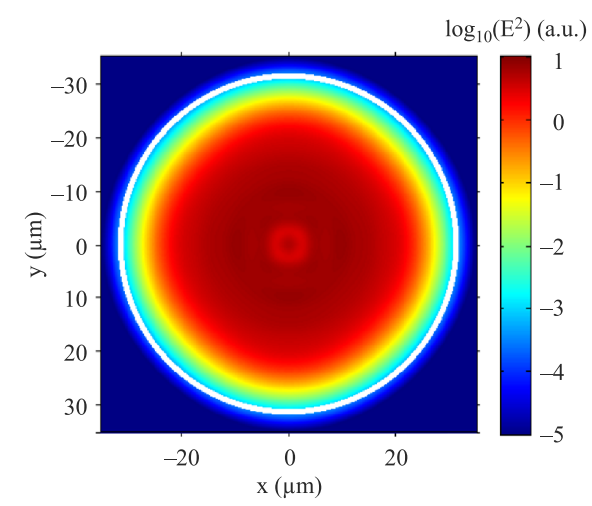

(d)

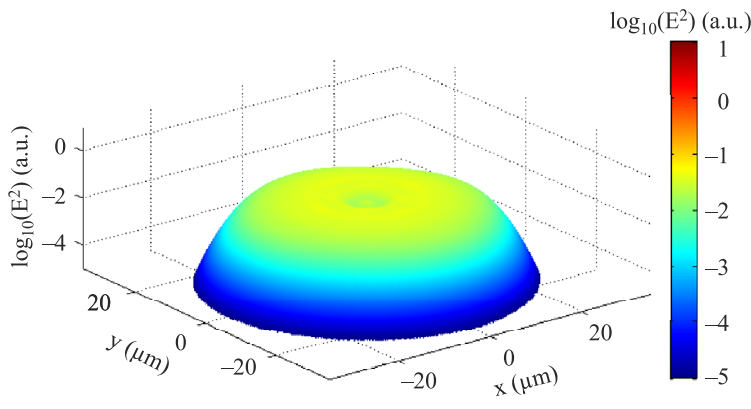

(c)

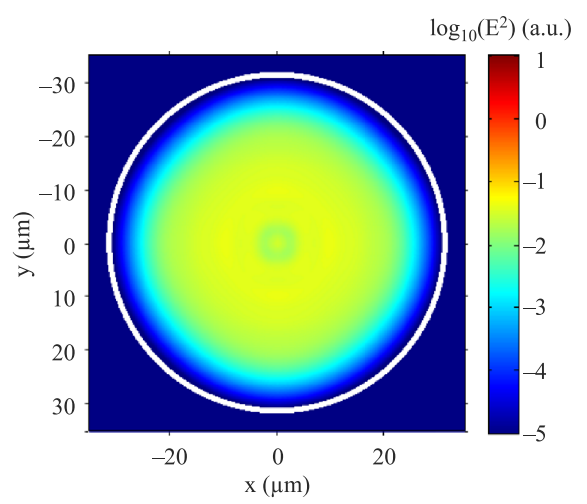

(e)

Fig. 2. Simulation of beam propagation through the microbridge: (a) intensity of light, (b) pseudo-3D image of intensity distribution of transmitted light, (c) pseudo-3D image of intensity distribution of reflected light, (d) 2D cross-section intensity distribution of transmitted light, and (e) 2D cross-section intensity of reflected light. Right bars represent the color scale of common logarithm of intensity in a.u. The dark contour lines in (a) are drawn for a 0.5 value change of intensity.

bottom of the fibre is different. It depends on the mode field diameter of the used fibres and can be fit to different cores' size of the connected fibres (microbridge with different width at two sides) by applying different optical power introduced to such fibres [19].

Optimization of the microbridge technology shows that time change of light exposition has small influence on the microbridge shape, but affects its mechanical strength. The process of photopolymerization arises rapidly and even $1 \mathrm{~s}$ is enough for obtaining a microbridge. However, such microbridge does not have good mechanical properties because of its irregularly polymerized structure. This situation is caused by a competitive reaction of the free radicals with oxygen. In order to obtain fully polymerized structure it is better to keep the photopolymerization longer. In Fig. 3 is shown transparency of a structure during the preparation of the polymer microbridge measured for the light beam at a wavelength of $633 \mathrm{~nm}$. As we can see, polymerization is a very fast process and the stabilization of the transmitted beam power occurred in the first seconds of the process. Longer time of exposition is only to ensure the whole mixture is fully polymerized on the path of the optical beam. For the above reason, after an experimental study, the optimum time equal to 60 seconds was selected, for the manufacture of polymer microbridges which work as converters between POF-SOF. Such a time guarantees full polymerization of the mixture and allows to produce the most durable polymer microbridge. 


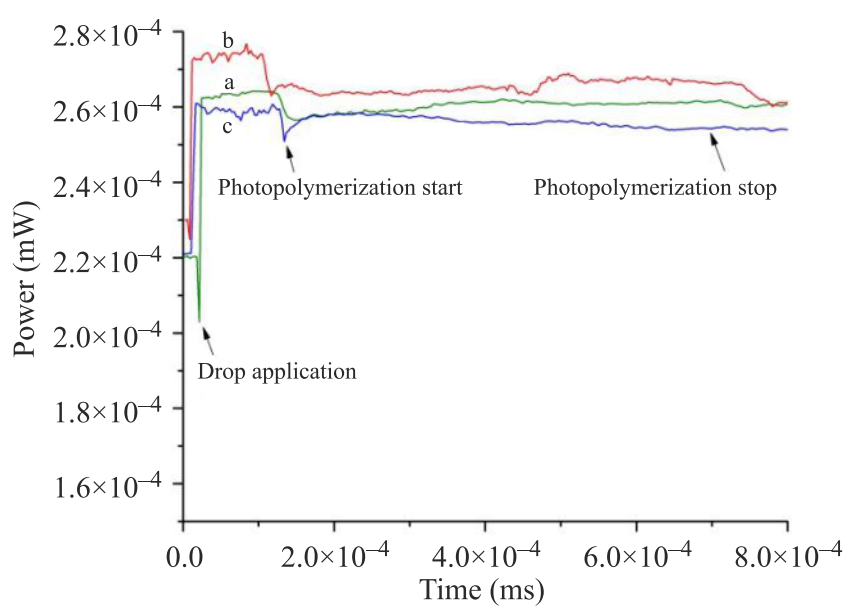

Fig. 3. Transparency of structure during the preparation of a polymer microbridge measured for the light beam at a wavelength of $633 \mathrm{~nm}$ for a POF-SOF connection. Tree characteristics named a, b, c are obtained during the preparation of microbridges with different mixtures.

In general, there is no technological restriction concerning the length of a microbridge - it can be about dozens of times longer than the core diameter as shown in experiments with polymer microtips $[11,22,23]$. Similar conclusions regarding long microbridge toughness to its bending and fibres axial misalignment were presented by Bachelot $e t$ al. [17]. But for optimized fibres' connections the length of a microbridge cannot be too long. Length of the element is dependent on the gap between the fibres and this parameter has been identified as the main one which influences converter optical losses. According to data presented in Fig. 4, for a microbridge manufactured at $5 \mu \mathrm{W}$ of incident light, the longer structure, the higher optical losses is obtained. Since the losses below $0.2 \mathrm{~dB}$ occur for the microbridges with the length up to $50 \mu \mathrm{m}$ and taking into consideration feasibility of a technological process (the minimum distance between

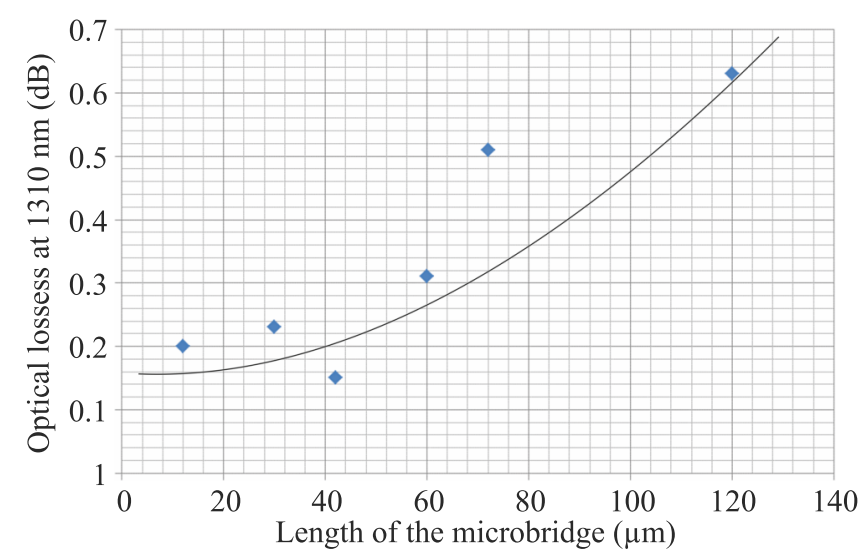

Fig. 4. Influence of the microbridge length on optical losses in a POF-SOF connection. fibres that enables maintaining photopolymerizable mixture in the gap between fibres), as well as future structure application as sensor elements [26,27], the optimal length has been chosen at $40 \mu \mathrm{m}$.

Finally, for the series of converters with the length of $40 \mu \mathrm{m}$ obtained with the output optical power of $5 \mu \mathrm{W}$ and a $60 \mathrm{~s}$ exposition time, the average optical losses for an optimized SOF-POF connection were of $0.20 \pm 0.03 \mathrm{~dB}$ at $1310 \mathrm{~nm}$. Additionally, as can be seen in Fig. 5, the optimization procedure for a microbridge between different size of optical fibres results in a structure with different widths on their two sides. This situation is caused by the different mode structure in POF and SOF where the mode spot size is higher for the POF structure. Since the connector optimization procedure needs the same optical power in two ends of the connected fibres, differences of mode structures manifest as a difference of microbridge width in two sides.

Stability of spectral characteristics for the obtained converter in a wide temperature range from $-40^{\circ} \mathrm{C}$ to $+80^{\circ} \mathrm{C}$ has been investigated using supercontinuum source and optical spectrum analyzer by placing manufactured structures in the climate chamber. MMFs has been used as a patch cord to a laser source and an optical analyzer, and thermal characteristics of the above fibre has been used as a reference for thermal investigation (Fig. 6). As can be seen from the data presented in Fig. 6, temperature does not cause changes in spectral characteristics and manufactured converters exhibit wave guiding properties in a wide spectral range. High attenuation at spectral characteristics in the entire range, around $10 \mathrm{~dB}$ for SOF-POF converter, is observed. This is associated with additional connectors between POF and SOF outside of the climate chamber necessary to connect the investigated structure with the instrumentation. It means, that the manufactured polymer converters can work properly in the temperature range of $-40^{\circ} \mathrm{C}+80^{\circ} \mathrm{C}$.

Finally, Figure 7(a) presents optical losses of the manufactured optimal polymer converter between POF and SOF at the wavelength of $\lambda=1310 \mathrm{~nm}$ in the temperature range

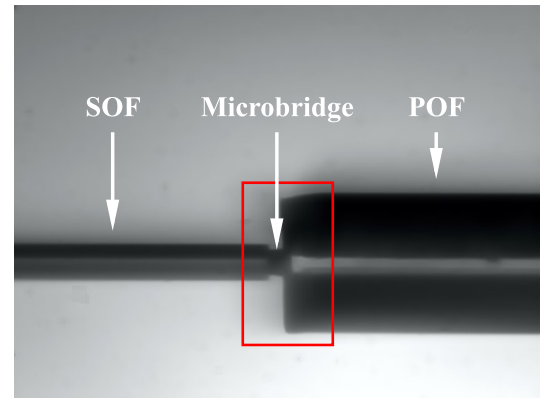

(a)

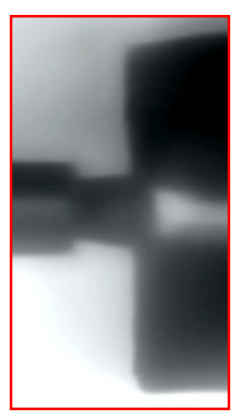

(b)
Fig. 5. Polymer converter manufactured between SOF-POF fibres (a) and magnitude area of the microbridge marked as a red rectangle (b). 


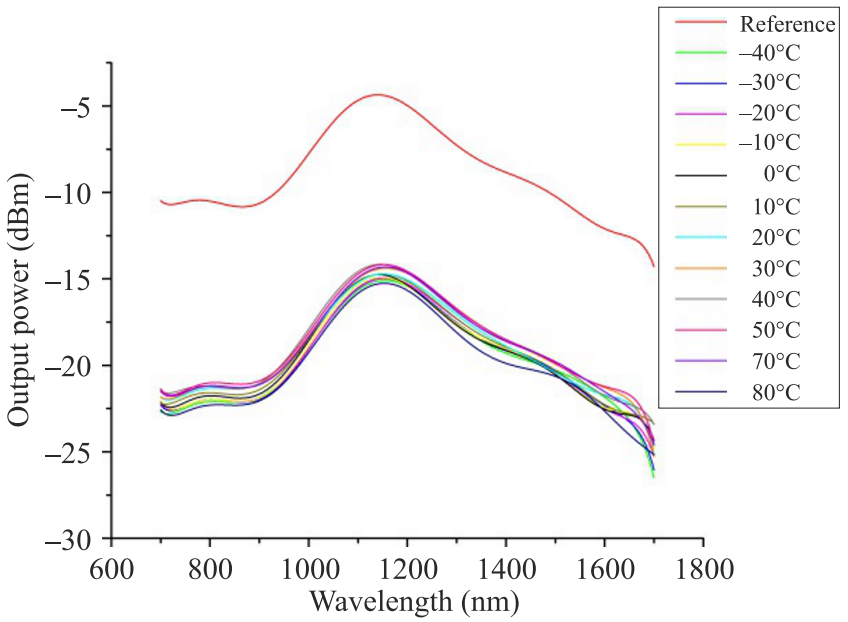

Fig. 6. Spectral characteristics depending on temperature for a SOF-POF converter.

form $-40^{\circ} \mathrm{C}$ to $+80^{\circ} \mathrm{C}$. As can be seen optical losses of a SOF-POF converter vary around the value of $0.3 \mathrm{~dB}$. These instabilities are connected with mode interaction in the structure, caused by mechanical vibrations of the climate chamber during changes of the temperature. Figure 7(b) presents the intensity distribution of first 20 modes propagated through the shifted converter. The microbridge was shifted by $-5 \mu \mathrm{m}$ in the $\mathrm{x}$ direction (to the left side of the presented image) while the second fibre (SOF) was shifted by $5 \mu \mathrm{m}$ in the $\mathrm{x}$ direction (to the right side of the presented image) relatively to the first fibre (POF). The difference between the normal beam distribution and the one for the shifted converter was presented in Fig. 7(c). Values shown in both Figs. 7(b) and 7(c) are in common logarithm of intensity. As one can see, from the simulation presented in Fig. 7(c), the small movement $(5 \mu \mathrm{m})$ of connected fibres generates difference in modal structure. It has a direct influence on the structure's optical losses, and, finally, the converter should be fixed in an external mechanical box for protection from such movements. However, the conducted research studies confirm that the polymer converter effectively connects polymer optical fibres with silica fibres in the wide temperature range. Moreover, after repeated heating and cooling of the polymer microbridge, optical losses do not increase. Hence, the thermal stress generated at the junction has little effect on the decrease in light transmitted power.

\section{Conclusions}

The aim of presented research has been the fabrication of waveguide polymer structures, called a converter, as permanent junctions for efficient coupling of polymer optical fibres with silica ones. Opto-mechanical differences between materials used for the above structures are the main difficulties for such connection. Application of classical splicing technology is impossible to use. The manufacturing of an optical microbridge as a waveguide between two optical fibres - silica fibre and polymer fibre, with the lowest possible optical losses seems to be a very interesting opportunity to solve the above technological problem. The process of photopolymerization is an easy and low cost method to obtain waveguide polymer structures. By the fact that a polymer microstructure grows with adaptation to a guided mode structure in an optical fibre, the proposed microbridge can work as a converter between two different optical fibres. The optimization of the polymer structure fabrication technology has been carried out by selection of mixture's time exposition to light and optical power of the incident light beam which allowed to obtain structures with the best transmission characteristics. Such microbridges can be manufactured within time of exposition of around $60 \mathrm{~s}$ with length of $40 \mu \mathrm{m}$ at the output power equal to $5 \mu \mathrm{W}$. Although we investigated time in period of $1 \mathrm{~s}$ to $80 \mathrm{~s}$, microbridge's length from $12 \mu \mathrm{m}$ to $120 \mu \mathrm{m}$ and output power from $1 \mu \mathrm{W}$ to $100 \mu \mathrm{W}$, the above parameters seem to be optimized for a connection of GIPOF62 with MMF taking into consideration the preferred diameter and mechanical strength of the microbridge. Optical losses of the manufactured optimal polymer converter between POF and SOF were $0.20 \pm 0.03$ $\mathrm{dB} @ 1310 \mathrm{~nm}$ and around $1.15 \pm 0.10 \mathrm{~dB} @ 1310 \mathrm{~nm}$ for a POF-POF connection. Optical losses were determined for the wavelength of $1310 \mathrm{~nm}$, but such microbridge structure can be used in the wide range of light spectrum as it does not change the light transmission. The idea of this paper is that a microbridge can be absolutely easily applied to other fibres without any changes in the set-up. From the above we believe that presented technology is suitable for connection of different kinds of fibres for instance with a large core diameter difference because microbridge width on both sides has a diameter equal to the mode field diameter of a given fibre. These are the first systematic results, to the best of our knowledge, regarding a manufacturing process of polymer microbridges between polymer optical fibres and silica ones. In authors' opinion, this is the best possible process for the preparation of a permanent junction between polymer and silica fibres.

\section{Acknowledgements}

The authors gratefully acknowledge support for this work from the National Research and Development Centre of Poland, especially under its Operative Programme Innovation Economy within key project POIG.01.03.01-14-016/08: New Photonic Materials and their Advanced Applications as well as the Polish Ministry of Science and Higher Education under MUT statutory activity PBS-654. 


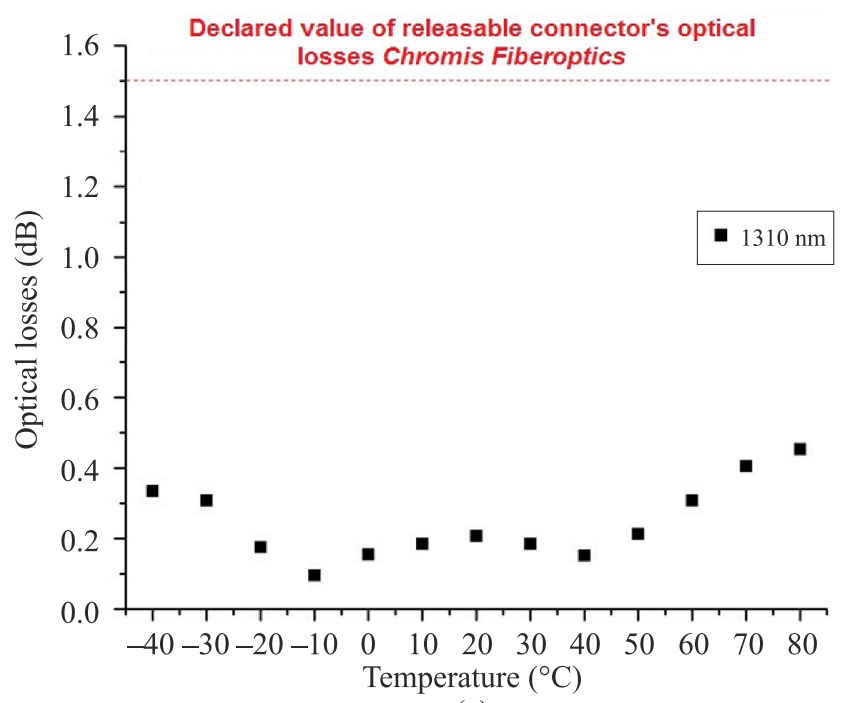

(a)

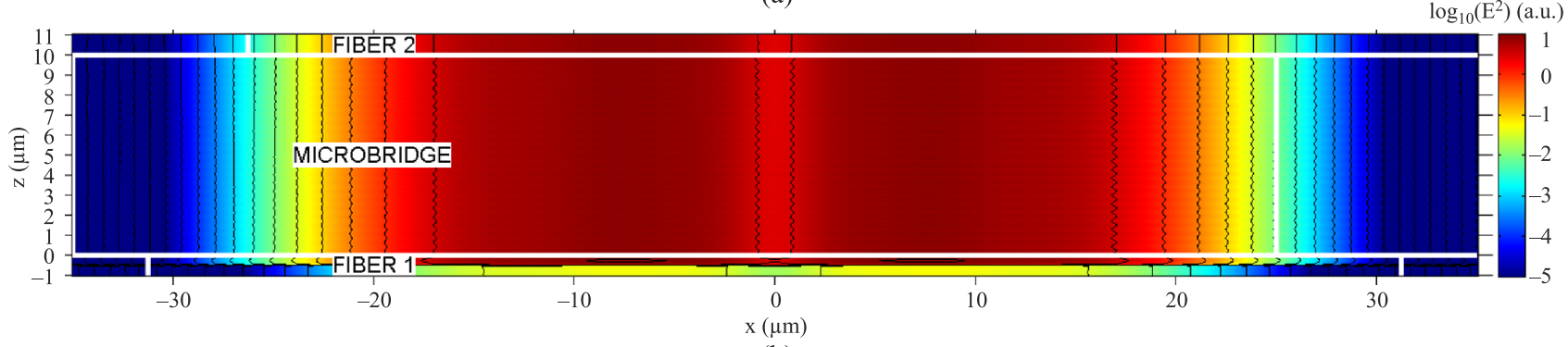

(b)

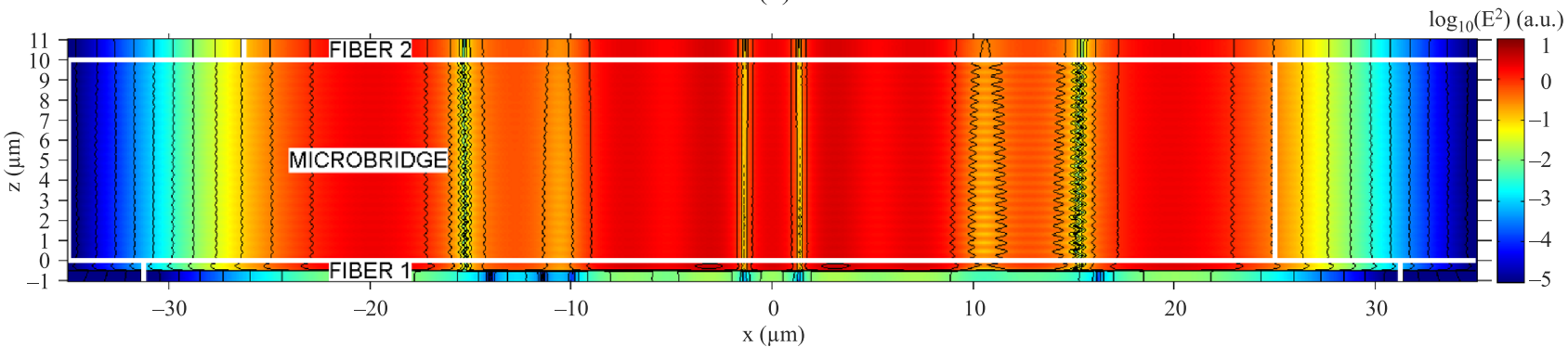

(c)

Fig. 7. (a) Changes of optical losses depending on temperature at the wavelength of $1310 \mathrm{~nm}$ for a SOF-POF converter, (b) intensity of beam propagating through the microbridge shifted by $5 \mu \mathrm{m}$, and (c) difference between normal converter and shifted one. Right bars represent the color scale of common logarithm of intensity in a.u. The dark contour lines in (b) and (c) are drawn for a 0.5 value change of intensity.

\section{References}

1. Z. Ran and Y. Rao, "Laser-machined all-fibre in-line tip pressure sensor", Proc. SPIE 7503, 75032X-1 (2009).

2. C.R. Liao, T.Y. Hu, and D.N. Wang, "Optical fibre Fabry-Perot interferometer cavity fabricated by femtosecond laser micromachining and fusion splicing for refractive index sensing”, Opt. Express 20, 22813-22818 (2012).

3. F. Causa, A. Aliberti, A.M. Cusano, E. Battista, and P.A. Netti, "Supramolecular spectrally encoded microgels with double strand probes for absolute and direct miRNA fluorescence detection at high sensitivity", J. Am. Chem. Soc. 137, 1758-1761 (2015).
4. A. Ricciardi, A. Aliberrti, M. Giaquinto, A Micco, and A. Cusano "Microgel Photonics: a breathing cavity onto optical fibre tip", Proc. SPIE 9634, 963486-1 (2015).

5. K. Grattan and T. Sun, "Fibre optic sensor technology: an overview," Sensor Actuat. A-Phys. 82, 40-61 (2000).

6. P. Antunes M. Domingues, N. Alberto, and P. André, "Optical fibre microcavity strain sensors produced by the catastrophic fuse effect", IEEE Photon. Technol. Lett. 26, 78-81 (2014).

7. H.M. Presby, A.F. Benner, and C.A. Edwards, "Laser micromachining of efficient fibre microlenses", Appl. Opt. 29, 2692-2695 (1990). 
8. D. Kato, "Light coupling from a stripe-geometry GaAs diode laser into an optical fibre with spherical end", J. Appl. Phys. 44, 2756-2758 (1973).

9. L.G. Cohen and M.V. Schneider, "Microlenses for coupling junction lasers to optical fibres", Appl. Opt. 13, 89-94 (1974).

10. T. Shoji, T. Tsuchizawa, T. Watanabe, K. Yamada, and H. Morita, "Low loss mode size converter from $0.3 \mu \mathrm{m}$ square $\mathrm{Si}$ wire waveguides to singlemode fibres", IEEE Electron. Lett. 38, 1669-1670 (2002).

11. Z. Pan, H. Subbaraman, Y. Zou, X. Xu, X. Zhang, C. Zhang, Q. Li, L. J. Guo, and R.T. Chen "Quasi-vertical tapers for polymer-waveguide-based interboard optical interconnects", Photon. Res. 3, 317-323 (2015).

12. S. Zheng, X. Zeng, W. Luo, S. Jradi, J. Plain, M. Li, P. Renaud-Goud, R. Deturche, Z. Wang, J. Kou, R. Bachelot, and P. Royer, "Rapid fabrication of micro-nanometric tapered fibre lens and characterization by a novel scanning optical microscope with submicron resolution", Opt. Express 21, 30-38 (2013).

13. J. Zubia and J. Arrue, "Plastic optical fibres: an introduction to their technological processes and applications", Opt. Fibre Techn. 7, 101-140 (2001).

14. A. Acakpovi and P.L.M.V. Matoumona, "Comparative analysis of plastic optical fibre and glass optical fibre for home networks", IEEE 4th Int. Conf. Adaptive Science \& Technology, 154-157 (2012).

15. A.D. Yablon, Optical fibre fusion splicing, Springer, Berlin, New York, 2005.

16. K. Egashira and M. Kobayashi, "Optical fibre splicing with a low-power $\mathrm{CO}_{2}$ laser”, Appl. Opt. 16, 1636 (1977).

17. R. Bachelot, C. Ecoffet, D. Deloeil, P. Royer, and D.-J. Lougnot, "Integration of micrometer-sized polymer elements at the end of optical fibres by free-radical photopolymerization", Appl. Opt. 40, 5860-5871 (2001).

18. S. Jradi, O. Soppera, and D.J. Lougnot, "Fabrication of polymer waveguides between two optical fibres using spatially controlled light-induced polymerization", Appl. Opt. 47, 3987 (2008)

19. L.R. Jaroszewicz, P. Marć, and P. Pura-Pawlikowska, "Process for preparation polymer converter for connecting optical fibres and polymer converter for connecting optical fibres", Patent Application, PCT/IB2015/5059519, 10 ${ }^{\text {th }}$ Dec. 2015.

20. K. Dorkenoo, O. Crégut, L. Mager, F. Gillot, C. Carre, and A. Fort, "Quasi-solitonic behavior of self-written waveguides created by photopolymerization", Opt. Lett. 27, 1782-1784 (2002).

21. P. Pura, M. Szymanski, B. Wisniowski, M. Dudek, L.R. Jaroszewicz, M. Napierala, A. Spadlo, P. Marc, and M. Kujawin- ska, "Polymer microtips fabricated at the extremity of photonic crystal fibres", J. Material Sci. Eng. B 3, 749-758 (2013).

22. P. Pura, M. Szymanski, M. Dudek, L.R. Jaroszewicz, P. Marc, and M. Kujawinska, "Polymer microtips at different types of optical fibres as functional elements for sensing applications", J. Lightwave Techn. 33, 2398-2404 (2015).

23. O. Soppera, S. Jradi, and D. J Lougnot, "Photopolymerization with microscale resolution: Influence of the physico-chemical and photonic parameters", J. Polymer Science Part A: Polymer Chemistry 46, 3783-3794 (2008).

24. S.N. Mensov and Yu.V. Polushtaytsev, "Coupling of fibres of different diameter in photopolymerizable composite", Optical Memory and Neural Networks 19, 61-68 (2010).

25. P. Pura, M. Szymanski, L.R. Jaroszewicz, P. Marc, M. Dudek, M. Kujawinska, M. Napierala, T. Nasilowski, and L. Ostrowski, "Microtips at photonic crystal fibres as functional elements for near-field scanning optical microscopy probes", Proc. SPIE, 9157, 91577U-91577U4 (2014).

26. L.R. Jaroszewicz, P. Marć, P. Pura, and I. Jakubowska, "Światłowodowy czujnik substancji chemicznych, zwłaszcza Bojowych Środków Trujących, w szczególności sarinu lub iperytu i ich pochodnych oraz sposób wytwarzania światłowodowego czujnika substancji chemicznych zwłaszcza Bojowych Środków Trujących, w szczególności sarinu lub iperytu i ich pochodnych", [in Polish], Patent Application P.408653, PPO 24th June 2014.

27. L.R. Jaroszewicz, P. Marć, P. Pura, and I. Jakubowska „Światłowodowy czujnik substancji chemicznych oraz sposób wytwarzania czujnika substancji chemicznych", [in Polish], Patent Application P.408652, PPO 24th June 2014.

28. O. Ziemann, J. Krauser, P. E. Zamzow, and W. Daum, POF- polymer optical fibres for data communication, Springer-Verlag, Berlin, 2002.

29. X. Zhang, A. Hosseini, X. Lin, H. Subbaraman, and R.T. Chen, "Polymer-based hybrid integrated photonic devices for silicon on-chip modulation and board-level optical interconnects", IEEE J. Selected Topics in Quantum Electronics 19, 3401115, (2013).

30. http://www.lumerical.com/.

31. A.W. Snyder and J. Love, Optical waveguide theory, Springer-Verlag, Berlin, 1984.

32. P. Pura, B. Wiśniowski, A. Spadło, L.R. Jaroszewicz, P. Marć, and M. Kujawińska, "Polymer microtips fabricated at the extremity of different type optical fibres", 4th Int. Conf. Metamaterials, Photonic Crystals and Plasmonics META'13, Sharjah, UAE, 2013. 